\title{
Adaptive Robust Maximum Power Point Tracking Control for Perturbed \\ Photovoltaic Systems with Output Voltage Estimation
}

\begin{abstract}
The problem of maximum power point tracking (MPPT) in photovoltaic (PV) systems, despite the model uncertainties and the variations in environmental circumstances, is addressed. Introducing a mathematical description, an adaptive sliding mode control (ASMC) algorithm is first developed. Unlike many previous investigations, the output voltage is not required to be sensed and the upper bound of system uncertainties and the variations of irradiance and temperature are not required to be known. Estimating the output voltage by an update law, an adaptive-based $\mathrm{H}_{\infty}$ tracking algorithm is then developed for the case the perturbations are energy-bounded. The stability analysis is presented for the proposed tracking control schemes, based on the Lyapunov stability theorem. From a comparison viewpoint, some numerical and experimental studies are also presented and discussed.
\end{abstract}

Keywords: environmental disturbance, robust tracking, voltage estimation, photovoltaic array, MPPT

\section{Introduction}

Photovoltaic systems have been widely utilized in various applications $[1,2]$ to convert the solar energy, as one of the main renewable energy sources, to electrical power. Due to such advantages as easy maintenance and availability of sunlight, the demand of PV power generation systems for both the industrial and residential applications have been increased in recent years. On the other hand, high installation cost of PV systems and low efficiency during rapid changing in environmental conditions may restrict the extensive utilization. To increase the efficiency of such systems, various investigations have been carried out in three 
main areas as (i) designing sun tracking systems [3], (ii) implementing effective power converter topologies, and (iii) developing MPPT algorithms [4]. The first two strategies are commonly adopted in designing and implementing the new PV systems, whereas developing MPPT schemes can be easily incorporated into the both new and installed systems. In fact, the MPPT problem is to adjust the PV operating point such that the solar power, delivered by the PV system, is maximized. In the presence of modeling errors, electrical noise, external disturbances, and model parameter variations, designing an effective MPPT scheme is inevitable to ensure robust accurate tracking. The algorithms as activation feedback control [5] or linear separation scheme [6] may not ensure the robustness properties. On the contrary, the adaptive-based strategies [7] and sliding control [8,9] may be more efficient for some classes of uncertain systems. Depending on the restrictions made on the system, various tracking control may be adopted to solve the MPPT problem.

The MPPT methods, may be classified to indirect ones, e.g., short-circuit and open-circuit schemes [10] which need a priori evaluation of the PV panel, or direct strategies such as perturb and observe [11] and incremental conductance, which operate at any meteorological conditions. The techniques may be fixed-step or variable-step [12], based on the methods by which the command variable is changed. Dealing with the system uncertainties, intelligent based methods as fuzzy logic [13, 14], and artificial neural network [15] have been proposed, which may suffer from lack of universality. Robust tracking methods $[9,16]$ have been also developed to ensure robust stability and performance, despite the perturbations. Combination of classical strategies with evolutionary algorithms [17, 18] may facilitate obtaining some additional goals as fast convergence, removing some measurements, and tracking the global peak of multiple PV arrays. However, apart from the category of the method, designing an MPPT algorithm with both the simplicity and universality properties is still highly desired. 
Reducing the number of required sensors for implementing the MPPT algorithms may also improve the cost-benefit of PV power systems.

Removing some drawbacks of the previous works, the main contributions of this investigation are, (i) reducing the required sensors by estimating the output voltage by an adaptation mechanism, (ii) the bound of system uncertainties and environmental disturbances are not required to be known in the design procedure, and (iii) robust tracking performance is ensured, as shown analytically by using the Lyapunov stability theorem. To this end, a mathematical model is presented in section 2 and the tracking control problem is stated. In section 3, an adaptive sliding mode algorithm is first presented to solve the MPPT problem. Then, dealing with the energy bounded perturbations in the PV systems, an $H_{\infty}$-based adaptive control strategy is proposed to ensure the convergence of maximum power voltage (MPV) tracking error to zero. In section 4, a comparison study is made from both the analytical and numerical viewpoints. An experimental configuration is implemented in section 5 for further verifying the results. The concluding remarks and some ideas for the future investigations are given in section 6.

\section{System Description}

In general, a PV system commonly consists of three main parts including PV array, power converter and load, as schematically shown in Fig. 1.

Fig.1. (Placed here)

\subsection{Mathematical Modelling}

Representing the output voltage and current of the PV array, respectively by $v_{p v}$ and $i_{p v}$, the electrical behavior of a PV array with $n_{p}$ parallel cells and $n_{s}$ series cells is characterized by 
$i_{p v}=n_{p} i_{p h}-n_{p} i_{r s}\left(e^{q v_{p v} / n_{s} A K_{b} T}-1\right)$

in which $q=1.6 \times 10^{-19} \mathrm{C}$ denotes the charge of an electron, $K_{b}=1.3805 \times 10^{-23} \mathrm{~J} / \mathrm{K}$ is Boltzmann's constant, $A$ represents the ideality factor of $\mathrm{P}-\mathrm{N}$ junction, and $T$ is the cell temperature in Kelvin. In characteristic equation (1), the reverse saturation current $i_{r s}$ and light-generated current $i_{p h}$ are calculated respectively by

$i_{r s}=i_{o r}\left(\frac{T}{T_{r}}\right)^{3} e^{\frac{q E_{g}}{K_{b} A}\left(\frac{1}{T_{r}}-\frac{1}{T}\right)}$

and

$i_{p h}=\left(i_{s c r}+K_{l}\left(T-T_{r}\right)\right) \frac{\lambda}{100}$

where $T_{r}$ is the reference temperature, $i_{\text {or }}$ denotes the reverse saturation current at $T_{r}, E_{g}$ is the band-gap energy of the semiconductor, $i_{s c r}$ represents the short circuit cell current at the reference temperature and insolation, $K_{l}$ is a positive coefficient, and $\lambda$ stands for insolation in $m W / \mathrm{cm}^{2}$.

The current- voltage PV characteristic under different temperatures at fixed irradiance 100 $\mathrm{mW} / \mathrm{cm}^{2}$, and the PV characteristic under different irradiance levels at fixed temperature $298^{\circ} \mathrm{K}$ are typically plotted in Figs. 2 and 3 respectively.

In order to evaluate the power along various insolation and cell temperatures, the PV output power is calculated with multiplying the PV current $i_{p v}$ by the PV voltage $v_{p v}$, which yields

$P_{p v}=n_{p} i_{p h} v_{p v}-n_{p} i_{r s} v_{p v}\left(e^{q v_{p v} / n_{s} A K_{b} T}-1\right)$

Figs. 2,3. (Placed here)

\subsection{MPPT control problem}

From a practical viewpoint, the environmental circumstances, e.g., the cell temperature $T$ and solar radiation $\lambda$ vary during the time. The power-voltage characteristic diagrams, typically 
plotted in Fig. 4 and 5, show that the maximum power is changed along various irradiance and cell temperatures. On the other hand, equations (1)-(4) show the dependence of the maximum power output voltage $v_{p v}^{*}$, at which the PV power is maximized, to such parameters.

Figs. 4,5 (Placed here)

The reference voltage $v_{p v}^{*}$ may be obtained by various search-based algorithms. Such point may be calculated analytically by solving $\frac{\partial P_{p v}}{\partial v_{p v}}=0$, which yields

$n_{p} i_{p h}-n_{p} i_{r s}\left(e^{\beta v_{p v}^{*}}-1\right)=n_{p} i_{r s} \beta v_{p v}^{*} e^{\beta v_{p v}^{*}}$

where $\beta=\frac{q}{n_{s} A K_{b} T}$ is defined for notational consistency.

The MPPT problem is to design a control signal such that the desired MPV $v_{p v}^{*}$ is tracked, despite the variations in the cell temperature and solar radiation or equivalently, the maximum solar power is delivered. To this end, use the Kirchhoff's current law in Fig. 1 to obtain

$\dot{v}_{p v}=\frac{1}{C_{p v}}\left(i_{p v}-i_{L}\right)$

where $i_{L}$ denotes the current through the inductance $L$. Moreover, by using the average method, the dynamic equations of the boost converter, is written as

$$
\begin{aligned}
& \dot{i}_{L}=\frac{1}{L} v_{p v}-\frac{1}{L} v_{o}(1-u)+\delta(t) \\
& \dot{v}_{o}=-\frac{1}{R C_{o}} v_{o}+\frac{1}{C_{o}} i_{L}(1-u)
\end{aligned}
$$

in which $u \in[0,1]$ denotes the duty cycle of the PWM control input, $v_{o}$ is the output voltage, and $\delta(t)$ stands for the system uncertainties and the variations in $v_{p v}$, arise due to possible changes in cell temperature and insolation. 


\section{Robust Adaptive MPPT Control Design}

As a preliminary step to design procedure, define the voltage and current tracking errors, respectively as

$$
e_{1}=v_{p v}-v_{p v}^{*}
$$

and

$$
e_{2}=i_{L}-i_{L}^{*}
$$

in which the reference current is adopted here as $i_{L}^{*}=i_{p v}-C_{p v} \dot{v}_{p v}^{*}$. Hence, the error dynamics are obtained as

$$
\begin{aligned}
& \dot{e}_{1}=\frac{1}{C_{p v}}\left(i_{p v}-i_{L}\right)-\dot{v}_{p v}^{*}=-\frac{1}{C_{o}} e_{2} \\
& \dot{e}_{2}=\frac{1}{L} v_{p v}-\frac{1}{L} v_{o}(1-u)+\delta(t)-i_{L}^{*}
\end{aligned}
$$

in which $\delta(t)$ is bounded by the positive constant $\eta$, i.e., $|\delta(t)|<\eta$. Meanwhile, an error metric function may be defined as

$S=e_{2}-\alpha e_{1}$

with $\alpha>0$.

Remark 1. The conservative assumption of measuring output voltage $v_{o}$ to construct the tracking control law, adopted in many previous works $[9,16]$, is relaxed here.

Remark 2. In the rest of paper, $\|V\|$ denotes the Euclidean vector norm for an $n \times 1$ vector $V$. Furthermore, $V \in L_{2}[0, T]$ if $\int_{0}^{T}\|V(t)\|^{2} d t<\infty, T \in[0, \infty)$, and $V \in L_{\infty}[0, \infty)$ if $\|V(t)\|<$ $\infty$ for all $t \in[0, \infty)$.

\subsection{Adaptive Sliding Mode Controller}

In order to develop an adaptive-based sliding mode MPPT control, take a sliding function as (13). In sliding mode control, the conditions $S=0$ and $\dot{S}=0$ should be satisfied $[9,20,21]$. 
Defining $\theta=\frac{1}{L} v_{o}$, as an unknown parameter to be estimated by the proposed control algorithm, the error dynamic (12) is rewritten as

$\dot{e}_{2}=\frac{1}{L} v_{p v}-\dot{i}_{L}^{*}-\theta(1-u)+\delta(t)$

The ASMC problem is to propose the control input $\mathrm{u}$

Theorem 1. Consider the dynamical equations of a photovoltaic power generation system, formed by (6)-(8). The control input

$u=\frac{1}{\hat{\theta}}\left(-\frac{1}{L} v_{p v}+i_{L}^{*}-\frac{\alpha}{C_{o}} e_{2}+\hat{\theta}-K \operatorname{sgn}(S)\right)$

in which $K>\eta$, together with the update law

$\dot{\hat{\theta}}=-\gamma_{1}(1-u) S, \quad \hat{\theta}(0)>0$,

where $\gamma_{1}>0$ denotes the adaptation gain, ensures maximum power point tracking.

Proof. Take the Lyapunov function candidate

$V(S, \tilde{\theta})=\frac{1}{2} S^{2}+\frac{1}{2 \gamma_{1}} \tilde{\theta}^{2}$

where $\tilde{\theta}=\theta-\hat{\theta}$ denotes the estimation error. Taking the time derivative of $V(S, \tilde{\theta})$ gives

$\dot{V}(S, \tilde{\theta})=S \dot{S}+\frac{1}{\gamma_{1}} \tilde{\theta} \dot{\tilde{\theta}}$

in which $\dot{S}$ can be obtained by using (11) and (14) as

$\dot{S}=\dot{e}_{2}-\alpha \dot{e}_{1}$

$=\frac{1}{L} v_{p v}-i_{L}^{*}-\tilde{\theta}(1-u)-\hat{\theta}(1-u)+\frac{\alpha}{C_{o}} e_{2}+\delta(t)$

Applying the control input (15) in (19) yields

$\dot{S}=-\tilde{\theta}(1-u)-K \operatorname{sgn}(S)+\delta(t)$

In order to calculate $\dot{V}(S, \theta)$, substitute (20) into (18) to obtain

$\dot{V}(S, \tilde{\theta})=-\tilde{\theta}(1-u) S-K|S|+\delta(t) S-\frac{1}{\gamma_{1}} \tilde{\theta} \dot{\hat{\theta}}$ 


$$
<-K|S|+\eta|S|-\frac{1}{\gamma_{1}} \tilde{\theta}\left(\dot{\hat{\theta}}+\gamma_{1}(1-u) S\right)
$$

By replacing the adaptation law (16) and choosing $K>\eta$, one can conclude $\dot{V}(S, \tilde{\theta})<0$ and consequently, the Lyapunov stability theorem [20] ensures the convergence of sliding surface $S$ to zero.

Now, replacing $e_{2}=-C_{o} \dot{e}_{1}$, obtained by (11), in sliding function (13) yields

$S=-C_{o} \dot{e}_{1}-\alpha e_{1}=0$

which represents a stable dynamic and the convergence of $e_{1}$ to zero is then concluded, i.e., the MPV tracking is guaranteed. Meanwhile, the current error $e_{2}$ also tends to zero, as described by a linear combination of $e_{1}$ and $S$ in (13), i.e., $e_{2}=S+\alpha e_{1}$.

Remark 3. In practice, adopting the upper bound $\eta$ in controller design may be a conservative, as it varies from one ambient condition to another and violates the universality of the method.

Theorem 2. For a PV system, described by (6)-(8) and subjected to any bounded perturbation $\delta(t)$, an adaptive sliding control algorithm, formed by (15) and (16), in which $K$ is obtained by adaptation mechanism

$\dot{K}=\gamma_{2}|S|$

solves the MPPT problem, without any knowledge about the bound of perturbations.

Proof. Choose a Lyapunov function as

$V(S, \widetilde{\theta}, \widetilde{K})=\frac{1}{2} S^{2}+\frac{1}{2 \gamma_{1}} \widetilde{\theta}^{2}+\frac{1}{2 \gamma_{2}} \widetilde{K}^{2}$

where $\widetilde{K}=\eta-K$ denotes the bound estimation error. To calculate $\dot{V}(S, \widetilde{\theta}, \widetilde{K})$ as

$\dot{V}(S, \tilde{\theta}, \widetilde{K})=S \dot{S}+\frac{1}{\gamma_{1}} \tilde{\theta} \tilde{\theta}+\frac{\dot{1}}{\gamma_{2}} \widetilde{K} \dot{\widetilde{K}}$

use (20) to obtain 


$$
\begin{aligned}
\dot{V}(S, \tilde{\theta}, \widetilde{K}) & =-\tilde{\theta}(1-u) S-K|S|+\delta(t) S-\frac{1}{\gamma_{1}} \tilde{\theta} \dot{\hat{\theta}}-\frac{1}{\gamma_{2}} \widetilde{K} \dot{K} \\
& <-K|S|+\eta|S|-\frac{1}{\gamma_{2}} \widetilde{K} \dot{K}-\tilde{\theta}(1-u) S-\frac{1}{\gamma_{1}} \tilde{\theta} \dot{\hat{\theta}}
\end{aligned}
$$

Incorporating update laws (16) and (23) into (26) gives $\dot{V}(S, \widetilde{\theta}, \widetilde{K})<0$, and through a procedure, similar to proof of Theorem 1, the convergence of $e_{1}$ and $e_{2}$ is concluded.

Remark 4. Avoiding the chattering phenomenon in sliding mode control, the sgn function may be replaced by a saturation function [20] or some approximation terms as

$\operatorname{sgn}(S) \approx \frac{S}{|S|+\sigma e^{-r t}}$

with $\sigma>0$ and $r>0$.

\subsection{Adaptive $H_{\infty}$ Tracking Control}

From a disturbance attenuation viewpoint, for any perturbation $\delta(t)$ with unknown bound, an adaptive-based $H_{\infty}$ control is proposed here to ensure the robustness property in the MPPT algorithm.

Theorem 3. For the perturbed PV system formed by (6)-(8), the control law

$u=\frac{1}{\hat{\theta}}\left(-K_{f} S-\frac{1}{L} v_{p v}+i_{L}^{*}-\frac{\alpha}{C_{o}} e_{2}+\hat{\theta}-\frac{1}{2 \rho} S\right)$

where $K_{f}$ is a positive gain and $\rho>0$ is a prescribed attenuation level, with adaptation mechanism (16) ensures that

(i) the MPP tracking error is uniformly ultimately bounded.

(ii) the $H_{\infty}$ tracking performance

$K_{f} \int_{0}^{T} S^{2}(t) d t \leq I_{0}+\frac{1}{2} \rho \int_{0}^{T}|\delta(t)|^{2} d t$ 
where $I_{0}>0$ depends to the initial condition, is achieved and the tracking error is converged to zero, if the perturbation is energy bounded, i.e., $\delta(t) \in L_{2}$.

Proof. Take the Lyapunov function (17). To calculate the time derivative $\dot{V}(S, \tilde{\theta})$ as (18), first incorporate the control input (28) into (19) to obtain

$\dot{S}=-K_{f} S-\tilde{\theta}(1-u)-\frac{1}{2 \rho} S+\delta(t)$

Substituting (30) into (18) with some manipulations gives

$$
\begin{aligned}
\dot{V}(S, \tilde{\theta}) & =-K_{f} S^{2}-\tilde{\theta}(1-u) S-\frac{1}{2 \rho} S^{2}+\delta(t) S-\frac{1}{\gamma_{1}} \tilde{\theta} \dot{\hat{\theta}} \\
& =-K_{f} S^{2}-\frac{1}{2 \rho}(S-\rho \delta)^{2}+\frac{1}{2} \rho \delta^{2}-\frac{1}{\gamma_{1}} \tilde{\theta}\left(\dot{\hat{\theta}}+\gamma_{1}(1-u) S\right)
\end{aligned}
$$

Now, applying the adaptation mechanism (16) in (31) yields

$\dot{V}(S, \tilde{\theta}) \leq-K_{f} S^{2}+\frac{1}{2} \rho|\delta|^{2}$

The following consequents are then concluded.

(i) By inequality (32), $\dot{V}(S, \tilde{\theta})$ can be bounded as $\dot{V}<-K_{f} S^{2}+\frac{1}{2} \rho \eta^{2}$. By choosing $K_{f}>$ $\frac{\rho \eta^{2}}{2 \epsilon}$, for any $\epsilon>0$, there exists a $\kappa>0$ such that, $\dot{V}<-\kappa S^{2}$ for all $|S| \geq \epsilon$. Hence, there is a bounded time as $T>0$ which implies $|S|<\epsilon$ for all $t \geq T$, i.e., the error metric function $S$ in uniformly ultimately bounded (UUB) [22]. By (11) and (13), one can write $S=-C_{o} \dot{e}_{1}-\alpha e_{1}$ to conclude that the MPP tracking error $e_{1}$ is also UUB.

(ii) Integrating the inequality (32) yields

$K_{f} \int_{0}^{T} S^{2}(t) d t+V(S(T), \tilde{\theta}(T)) \leq V(S(0), \tilde{\theta}(0))+\frac{1}{2} \rho \int_{0}^{T}|\delta(t)|^{2} d t$ for all $0 \leq T<\infty$, which implies the $H_{\infty}$ tracking performance (29) by defining $I_{0}=$ $V(S(0), \tilde{\theta}(0))-V(S(T), \tilde{\theta}(T))$ and consequently, $|S|$ is square-integrable, i.e., $S \in L_{2}$. On the other hand, the boundedness of all the closed loop signals in dynamic equation (30) along 
with control law (28) implies that $\dot{S}$ is also bounded, i.e., $\dot{S} \in L_{\infty}$. Using the proof, given for (i), the boundedness of $S$ is also concluded, i.e., $S \in L_{\infty}$, and the Barbalat's lemma [22] implies that $S$ is converge to zero. Meanwhile, the convergence of $e_{1}$ and $e_{2}$ is also concluded, following a procedure, similar to the proof of Theorem 1.

Remark 5. The reference point $\left(v_{p v}^{*}, i_{L}^{*}, v_{o}^{*}\right)$ may be regarded as the equilibrium point of the nonlinear dynamics (6)-(8). In fact, $v_{p v}^{*}$ is specified by (5), and solving the differential equations (7) and (8) at the steady state conditions yields $v_{o}^{*}=\sqrt{R i_{L}^{*} v_{p v}^{*}}$.

\section{Simulation Study}

The general structure of a PV system, depicted in Fig. 1, is constructed here with $L=$ $1.5 m H, C_{p v}=1000 \mu F, C_{o}=1000 \mu F$, and $R=25 \Omega$. The specifications of the adopted 60W PV module are given in Table 1.

Table 1 (Placed here)

To verify the effectiveness of the proposed adaptive-based MPPT algorithms, three cases are investigated here, in which a $10 \%$ deviation is assumed for the circuit elements.

Case 1. Fixed irradiance at $100 \mathrm{~mW} / \mathrm{cm}^{2}$ and cell temperature $25^{\circ C}\left(298^{\circ K}\right)$. The ASMC algorithm, formed by (16), (17), and (23) with $\alpha=2$ is applied here to solve the MPPT problem. Fig. 6 demonstrates the convergence of sliding surface $S(t)$, sliding gain $K$, and duty cycle $u(t)$. The time history of $i_{p v}, v_{p v}$, and PV generated power $P_{p v}$, as depicted in Fig. 7, shows that the maximum power $60 \mathrm{~W}$ is obtained in a short time. Moreover, applying the proposed ASMC scheme removes the necessity of output voltage measurement, by using the estimation of unknown parameter $\theta(t)$, as illustrated in Fig. 8. 
Figs. 6-8 (Place here)

Case 2. Trapezoidal variation in the cell temperature. In practice, a trapezoidal temperature profile may be adopted to evaluate the performance of the proposed MPPT algorithms, as the temperature changes during a day. By changing the temperature from $298{ }^{K}$ to $328{ }^{K}$ and applying the ASMC scheme, the MPV and the system responses are illustrated in Fig. 9. A comparison study, made by Fig. 10, shows that a faster response is achieved by the adaptivebased $H_{\infty}$ tracking control with $K_{f}=15$ and $\rho=0.1$, compared with the ASMC scheme. However, the robust convergence of system responses in the MPPT problem is ensured by the proposed methods.

Case 3. Trapezoidal change in the irradiance. Taking an standard irradiation profile which presents a trapezoidal variation, Fig. 11 shows that the ASMC ensures the robust convergence of the generated power to MPP. On the contrary, the performance of the proposed adaptive-based $H_{\infty}$ tracking control, may be compared by that of ASMC. As depicted in Fig. 12, the adaptive $H_{\infty}$ control algorithm not only presents a faster convergence in tracking error, but also gives a smoother control effort.

Removing the necessity of output measurement $[9,16]$, as a conservative assumption, fast response with implementable control efforts may be the other benefits of the methods, even in the presence of irradiance and temperature variations. The intelligent algorithms based on fuzzy logic and neural networks [13-15, 21, 23] may be also used to solve the MPPT problem, especially in steady-state conditions. Emphasizing on the robustness properties, fast convergence in transient conditions, and estimating the output voltage, this paper has focused on the adaptive-base robust algorithms.

Figs 9-12 (Placed here) 


\section{Experimental Verification}

An experimental set up is implemented to verify the performance of the proposed MPPT controller, in real world environment. The configuration of the system with a STP185S solar module from the Suntech company, and a picture of the system under test are reported in Fig. 13. The generated control effort is applied to MOSFET IRFP264, through the gate driver HCPL312. The experiment is conducted under the irradiance $484 \mathrm{w} / \mathrm{m}^{2}$ and temperature $20^{\circ C}$ $\left(293^{\circ K}\right)$, on Oct. 10,2015 . A $50 \Omega$ resistor is connected as load. The resulting duty cycle and the waveforms of applying the proposed ASMC algorithm are illustrated in Figs. 14. The power variation, mainly caused by low sampling rate, can be improved by high performance interfaces. Optimally integrating the circuit accessories may also facilitate developing a commercial package. Applying the proposed ASMC, the time history of PV current $i_{p v}$, and the PV generated power $P_{p v}$, may be compared with those of the existing $\mathrm{P} \& \mathrm{O}$ algorithm, by Fig. 15. In addition to the benefit of the proposed scheme in removing the necessity of output measurement, the higher mean generated power with lower fluctuations shows more total efficiency, compared with the $\mathrm{P} \& \mathrm{O}$ algorithm.

\section{Conclusions}

The variations in solar irradiance, cell temperature and system perturbations, form an uncertain mathematical model for a PV system. Removing the necessity of output voltage measurement, an adaptive sliding mode control is first presented to solve the underlying MPPT problem, in which the upper bound of perturbations is not required to be known. Then, an adaptive $H_{\infty}$ control algorithm is proposed to ensure the fast and robust tracking with smoother control input, as the environmental disturbances are energy-bounded in the underlying control problem. Ensuring the robust stability property by the Lyapunov stability theorem, the numerical studies are demonstrated to verify the robust tracking performance of 
the both algorithms. Although the steady state responses are almost identical for both of methods, the adaptive $H_{\infty}$ control algorithm presents faster transient response. Such evidence show that the $H_{\infty}$-based method may give more energy efficiency in varying environmental conditions, e.g., partial shading. From a comparison viewpoint, the experimental results are also presented to show the benefits of the proposed ASMC scheme, compared with an existing algorithm.

\section{Acknowledgment}

This work is supported by the Iran National Science Foundation (INSF), under research grant No. 91044147. We also would like to thank Ms. Marjan Faghih for her assistance in recording the experimental test results.

\section{Nomenclature}

$\begin{array}{llll}\lambda & \text { Solar insolation }\left(\mathrm{mW} / \mathrm{cm}^{2}\right) & C_{2} & \text { Output capacitance of converter } \\ T & \text { Cell temperature }(\mathrm{K}) & K_{b} & \text { Boltzmann's constant } \\ T_{r} & \text { Reference temperature }(\mathrm{K}) & E_{g} & \text { Semiconductor band-gap energy } \\ i_{p v} & \text { PV current (A) } & v_{p v} & \text { PV voltage (V) } \\ i_{p h} & \text { Light generated current (A) } & v_{o} & \text { Output voltage of converter } \\ i_{r s} & \text { Reverse saturation current (A) } & P_{p v} & \text { PV generated power } \\ i_{o r} & \text { Reverse saturation current at } T_{r} & C_{1} & \text { Input capacitance of converter } \\ i_{s c r} & \text { Short circuit current at } T_{r} & R & \text { Load resistance }\end{array}$




\section{References}

[1] Ghosh, A., Malla, S. G. and Bhende, C. N., 2015, "Small Signal Modelling and Control of Photovoltaic Based Water Pumping System,” ISA Transactions, 57, pp.382-389.

[2] Lajouad, R., Magri, A. E., Fadili, A. E., Chaoui, F.-Z. and Giri, F., 2014, "Adaptive Nonlinear Control of Wind Energy Conversion System Involving Induction Generator," Asian Journal of Control. doi: 10.1002/asjc. 1020.

[3] Mousazadeh, H., Keyhani, A., Javadi, A., Mobli, H., Abrinia, K., and Sharifi, A., 2009, “A Review of Principle and Sun-Tracking Methods for Maximizing Solar Systems Output", Renewable and Sustainable Energy Reviews, 13(8), pp.1800-1818.

[4] Kamarzaman, N. A., and Tan, C. W., 2014, "A Comprehensive Review of Maximum Power Point Tracking Algorithms for Photovoltaic Systems," Renewable and Sustainable Energy Reviews, 37, pp. 585-598.

[5] Wang X.Y., and Song, J.M., 2009, "Synchronization of the Fractional Order Hyperchaos Lorenz Systems with Activation Feedback Control," Communications in Nonlinear Science and Numerical Simulation, 14(8), pp. 3351-3357.

[6] Wang X.Y., and He Y., 2008, "Projective Synchronization of Fractional Order Chaotic System Based on Linear Separation," Physics Letters A, 372(4), pp. 435-441.

[7] Lin, D., Wang, X.Y., Nian, F., and Zhang, Y., 2010, "Dynamic Fuzzy Neural Networks Modeling and Adaptive Backstepping Tracking Control of Uncertain Chaotic Systems," Neurocomputing, 73(16-18), pp. 2873-2881.

[8] Wang, X.Y., Zhang, X., and Ma, C., 2012, "Modified Projective Synchronization of FractionalOrder Chaotic Systems via Active Sliding Mode Control," Nonlinear Dynamics, 69(1), pp. 511-517.

[9] Chu, C. C., and Chen, C. L., 2009, "Robust Maximum Power Point Tracking Method for Photovoltaic Cells: A Sliding Mode Control Approach," Solar Energy, 83, pp. 1370-1378.

[10] Masoum, M. A. S., Dehbonei, H., and Fuchs, E. F., 2002, "Theoretical and Experimental Analyses of Photovoltaic Systems with Voltage and Current Based Maximum Power-Point Tracking," IEEE Trans. Energy Conversion, 17, pp. 514-522.

[11] Femia, N., Petrone, G., Spagnuolo, G., and Vitelli, M., 2009, “A Technique for Improving P\&O MPPT Performances of Double Stage Grid-Connected Photovoltaic Systems," IEEE Trans. Industrial Electronics, 56(11), pp. 4473-82.

[12] Houssamo, I., Locment, F., and Sechilariu, M., 2013, "Experimental Analysis of Impact of MPPT Methods on Energy Efficiency for Photovoltaic Power Systems," Electrical Power and Energy Systems 46, pp. 98-107.

[13] Chui, C. S., "T-S Fuzzy Maximum Power Point Tracking Control of Solar Power Generation Systems,” 2010, IEEE Trans. Energy Conversion, 25(4), pp. 1123-1132.

[14] Rahim, N. A., Soh, A. C., Radzi, M. A. M., and Zainuri, M. A. A., 2014, "Development of Adaptive Perturb and Observe-Fuzzy Control Maximum Power Point Tracking for Photovoltaic Boost DC-DC Converter," IET Renewable Power Generation, 8(2), pp. 183-194. 
[15] Kharb, R. K. Shimi, S. L., Chatterji, S., and Ansari, M. F., 2014, "Modeling of Solar PV Module and Maximum Power Point Tracking Using ANFIS," Renewable and Sustainable Energy Reviews, 33, pp. 602-612.

[16] Chiu, C. S., Ouyang, Y. L., and Ku, C. Y., 2012, "Terminal Sliding Mode Control for Maximum Power Point Tracking of Photovoltaic Power Generation Systems," Solar Energy, 86, pp. 2986-2995.

[17] Liu, L., and Liu, C., 2012, "A Novel Combined Particle Swarm Optimization and Genetic Algorithm MPPT Control Method for Multiple Photovoltaic Arrays at Partial Shading," ASME Journal of Energy Resources Technology, 135(1), p. 012002.

[18] Tajuddin, M. F. N., Ayob, S. M., Salam, Z., and Saad, M. S., 2013, "Evolutionary Based Maximum Power Point Tracking Technique Using Differential Evolution Algorithm," Energy and Buildings, 76, pp. 245-252.

[19] Salas, V., Olias, E., Barrado, A., and Lazaro, A., 2009, "Review of the Maximum Power Point Tracking Algorithms for Stand-Alone Photovoltaic Systems," Solar Energy Materials \& Solar Cells, 90, pp. 1555-1578.

[20] Slotine, J. J. E., and Li, W., 1991, Applied Nonlinear Control, Prentice-Hall, New Jersey.

[21] Lin, D., and Wang, X.Y., 2010, "Observer-based Decentralized Fuzzy Neural Sliding Mode Control for Interconnected Unknown Chaotic Systems via Network Structure," Fuzzy Sets and Systems, 161(15), pp. 2066-2080.

[22] Krstic, M., Kanellakopoulos, I., Kokotovic, P., 1995, Nonlinear and Adaptive Control Design. Wiley, New York.

[23] Lin, D., and Wang, X.Y., 2011, "Self-organizing Adaptive Fuzzy Neural Control for the Synchronization of Uncertain Chaotic Systems with Random-Varying Parameters," Neurocomputing, 74(12-13), pp. 2241-2249.

Table 1. Specifications of the 60W PV module

\begin{tabular}{ll}
\hline Parameter & Value \\
\hline Reference Temperature $T_{r}$ & $298{ }^{\circ} \mathrm{K}$ \\
Reverse saturation current $i_{\text {or }}$ & $6 \times 10^{-8} \mathrm{~A}$ \\
Short circuit current $i_{s c r}$ & $3.75 \mathrm{~A}$ \\
Short circuit current coefficient $k_{l}$ & $2.4 \times 10^{-3} \mathrm{~A} /{ }^{\circ} \mathrm{K}$ \\
Band-gap energy $E_{g}$ & $1.1 \mathrm{eV}$ \\
Ideality factor $A$ & 1.2 \\
\hline
\end{tabular}




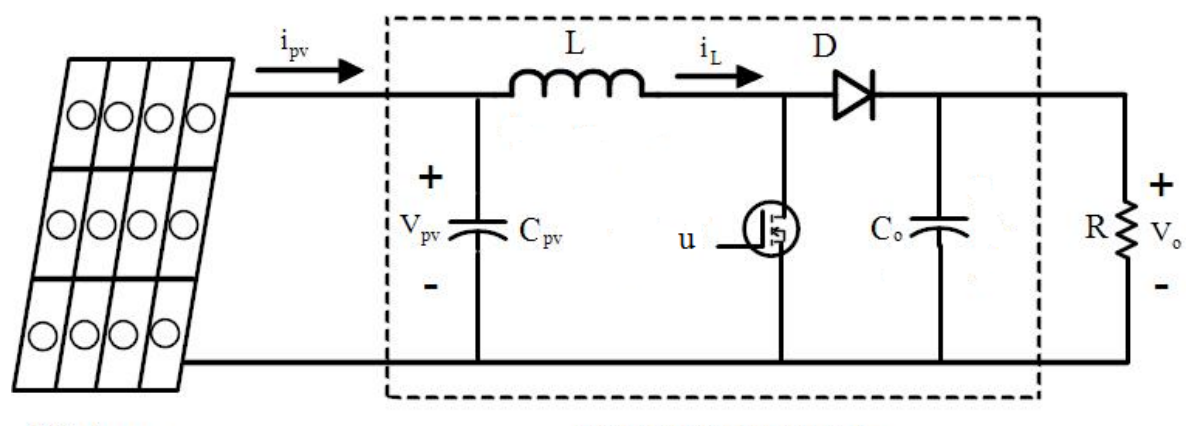

PV Array

DC-DC Boost Converter

Fig.1. Typical configuration of a PV system with a DC-DC boost converter

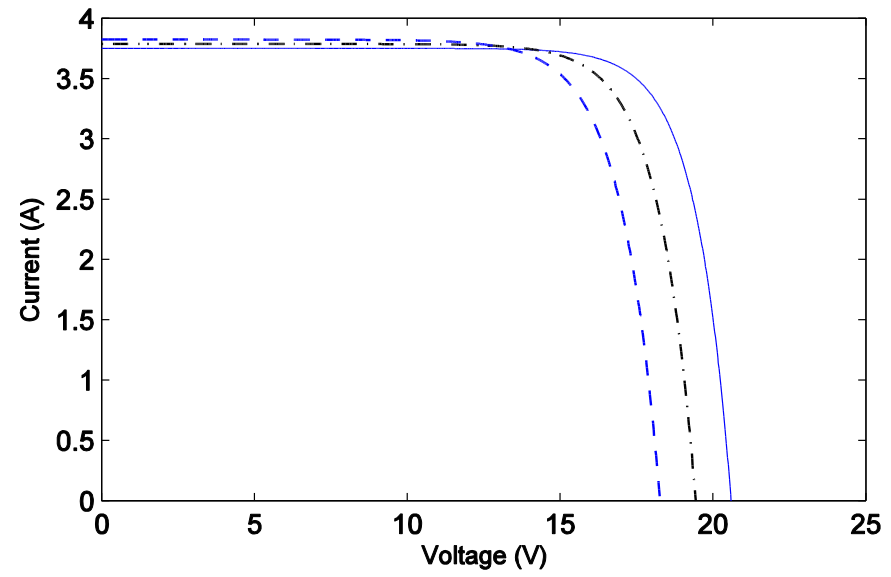

Fig. 2. PV characteristic diagram under the fixed irradiance $100 \mathrm{~mW} / \mathrm{cm}^{2}$ and different temperatures $298{ }^{\circ} \mathrm{K}$ (一), $313^{\circ} \mathrm{K}(-),. 328^{\circ} \mathrm{K}(--)$.

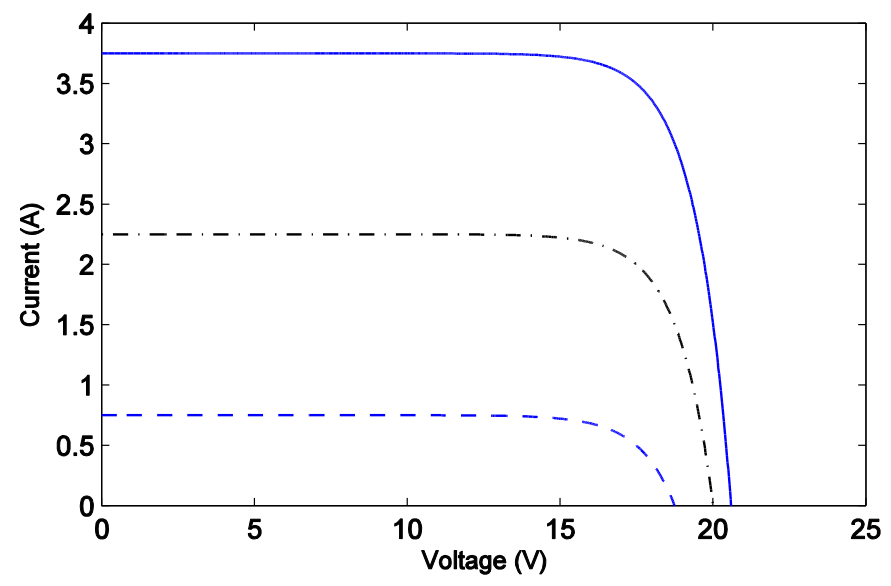

Fig. 3. PV characteristic diagram under the fixed temperature $298{ }^{\circ} \mathrm{K}$ and different irradiance levels 100 $\mathrm{mW} / \mathrm{cm}^{2}(-), 60 \mathrm{~mW} / \mathrm{cm}^{2}(-),. 20 \mathrm{~mW} / \mathrm{cm}^{2}(--)$. 


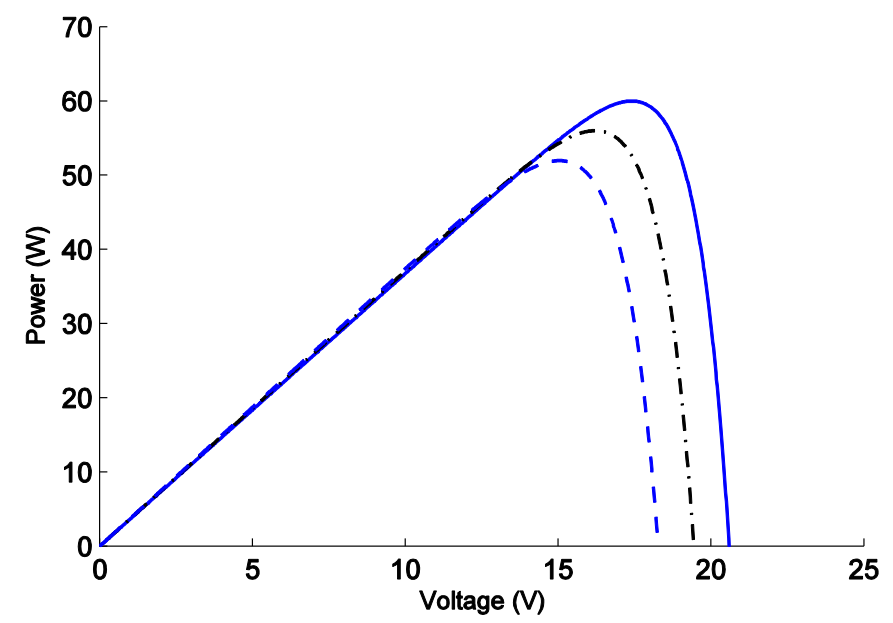

Fig. 4. Power-voltage characteristic curve under the fixed irradiance $100 \mathrm{~mW} / \mathrm{cm}^{2}$ and different temperatures $298{ }^{\circ} \mathrm{K}(-), 313^{\circ} \mathrm{K}(-),. 328^{\circ} \mathrm{K}(--)$

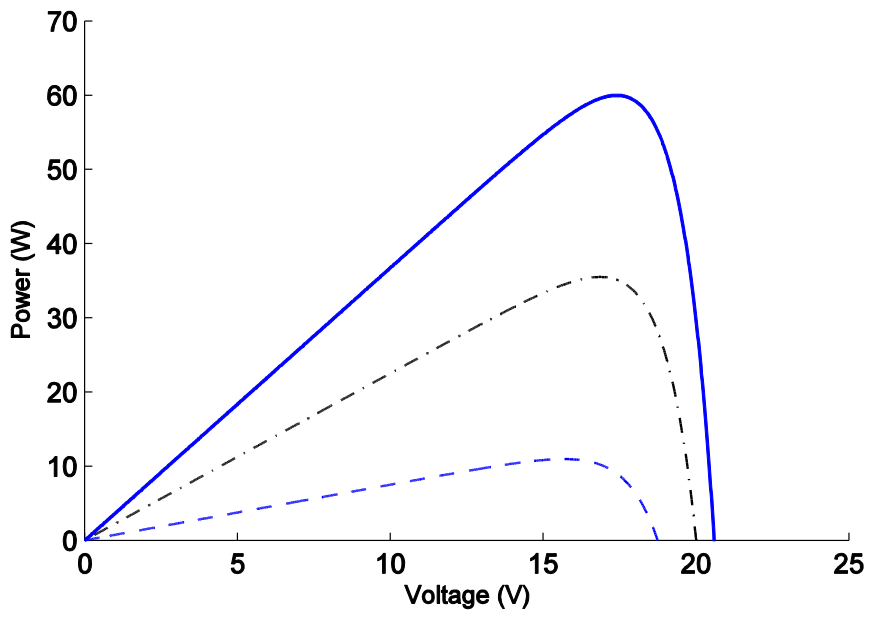

Fig. 5. Power-voltage characteristic curve under the temperature $298{ }^{\circ} \mathrm{K}$ and different irradiance levels 100 $\mathrm{mW} / \mathrm{cm}^{2}$ (-), $60 \mathrm{~mW} / \mathrm{cm}^{2}(-$ ) $), 20 \mathrm{~mW} / \mathrm{cm}^{2}(--)$ 


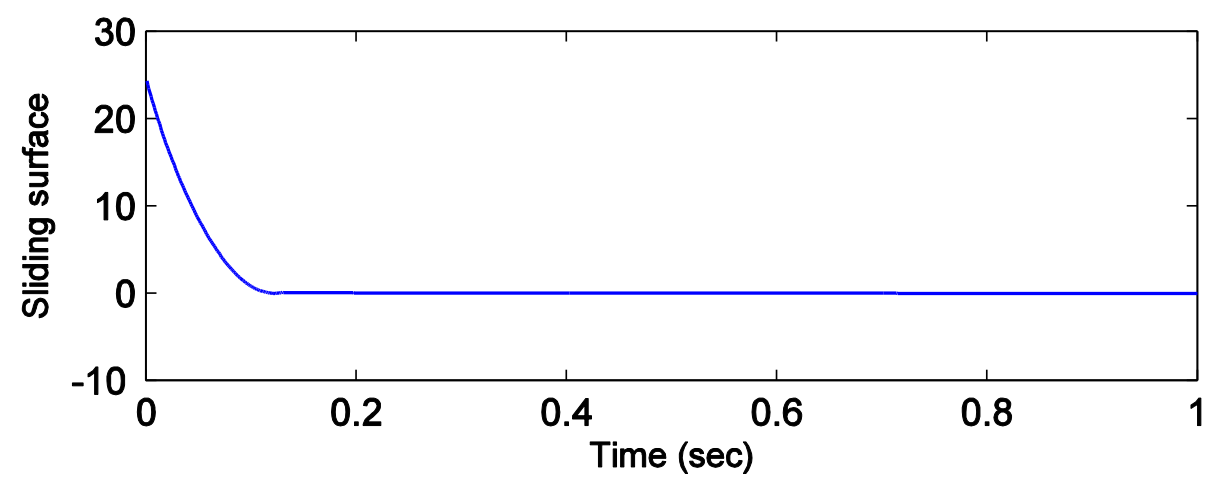

(a)

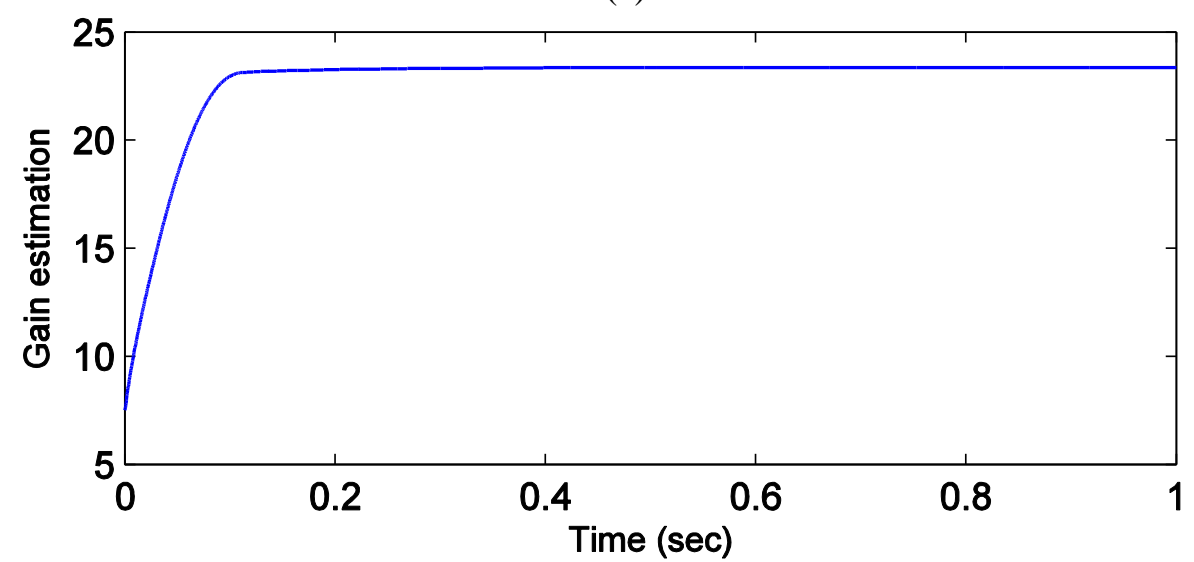

(b)

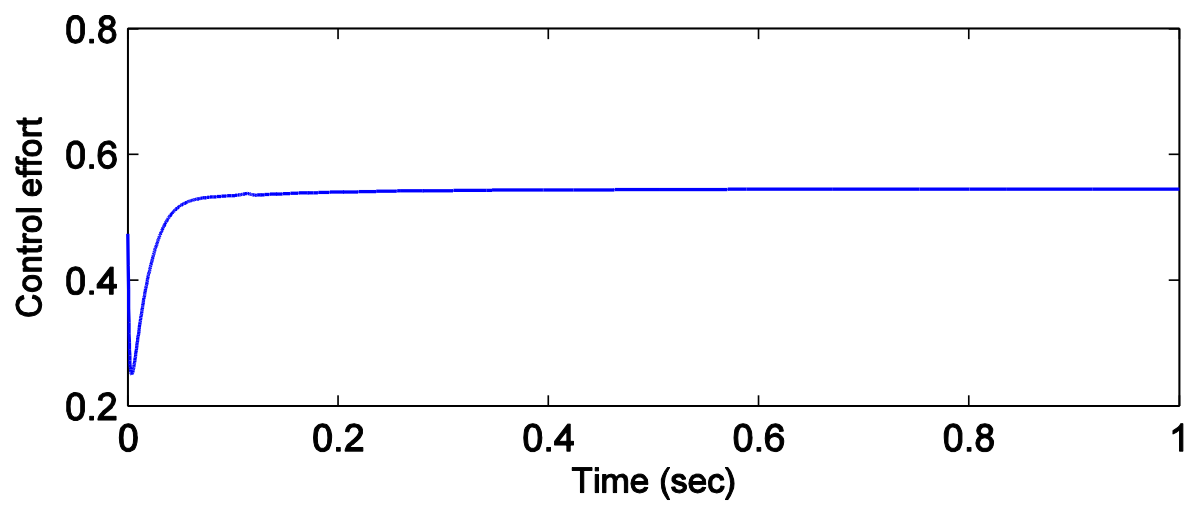

(c)

Fig. 6. MPPT control response in case 1, (a) Sliding surface $S(t)$, (b) Sliding gain estimation $K$, and (c) Control input $u(t)$.

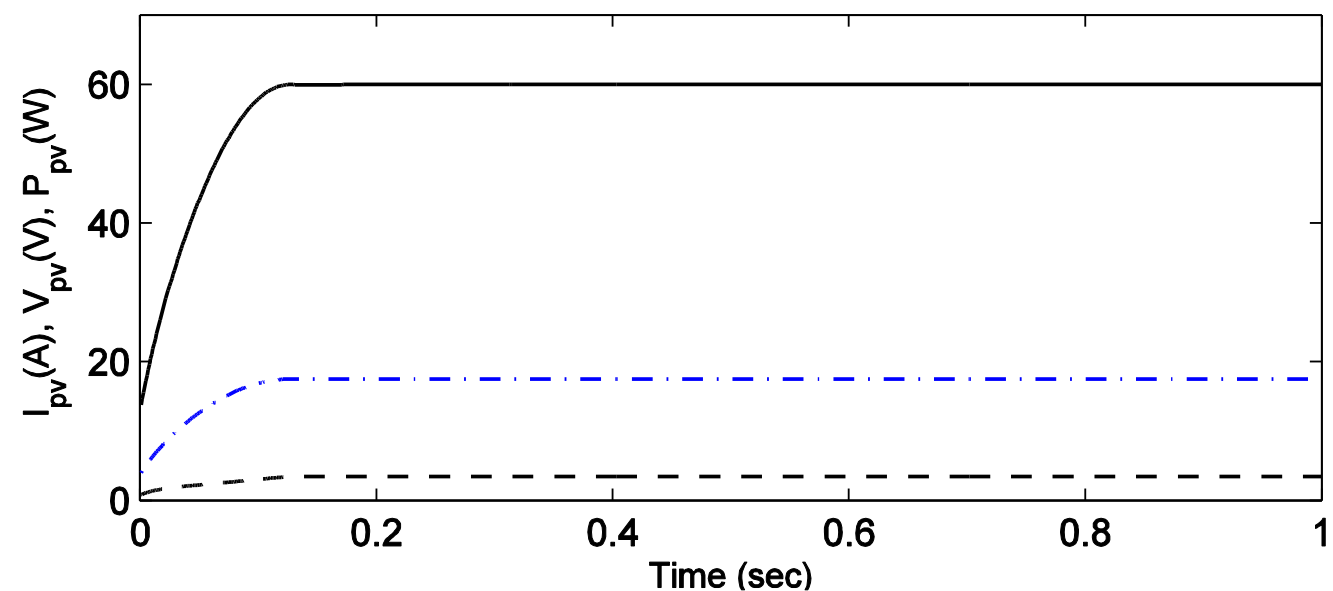

Fig 7. Time response of PV current $i_{p v}(--), \mathrm{PV}$ voltage $v_{p v}\left(-\right.$.), and PV generated power $P_{p v}(-)$, in case 1. 


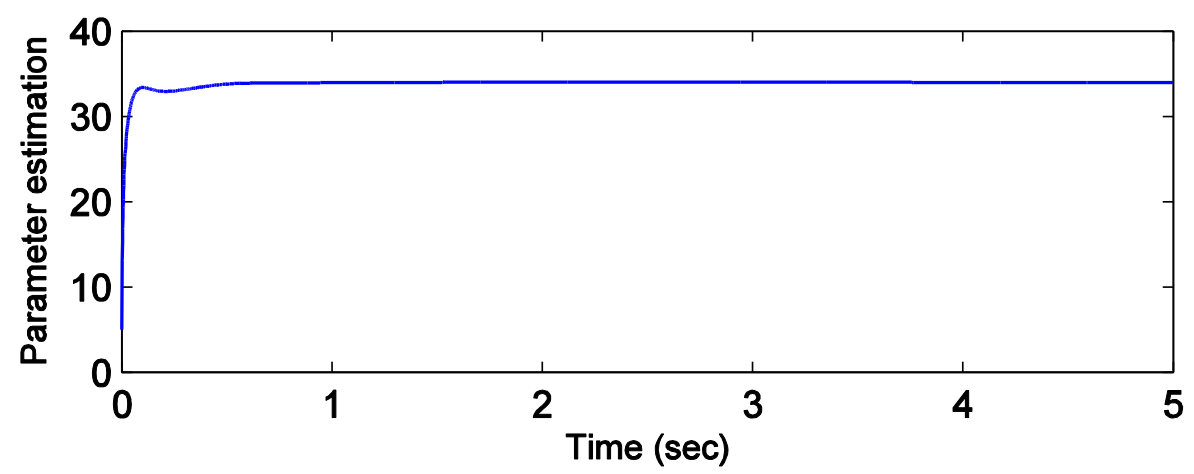

(a)

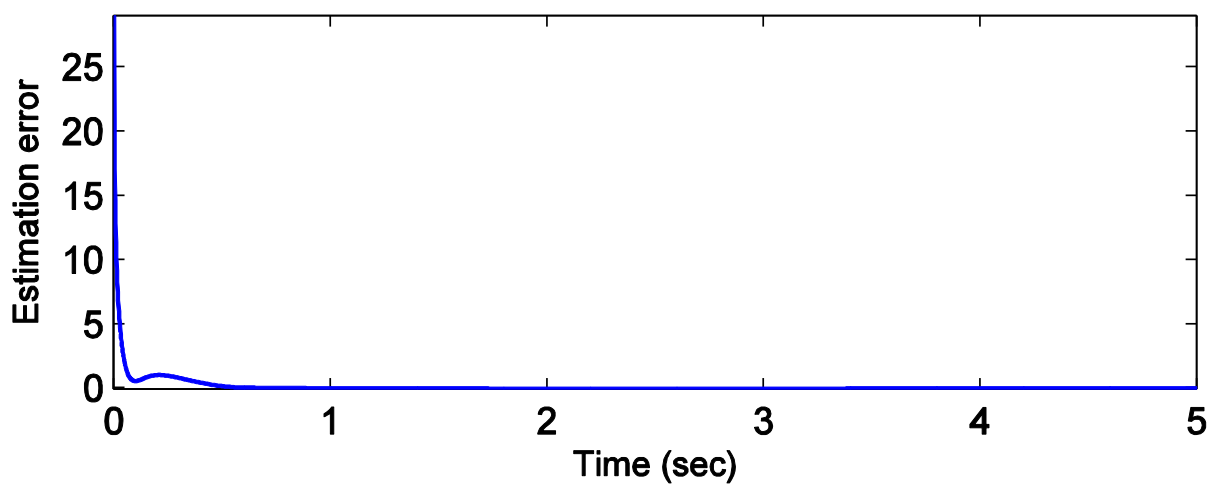

(b)

Fig. 8. Time history for estimation of $\theta$ in the ASMC algorithm, (a) Parameter estimation, and (b) Estimation error. 


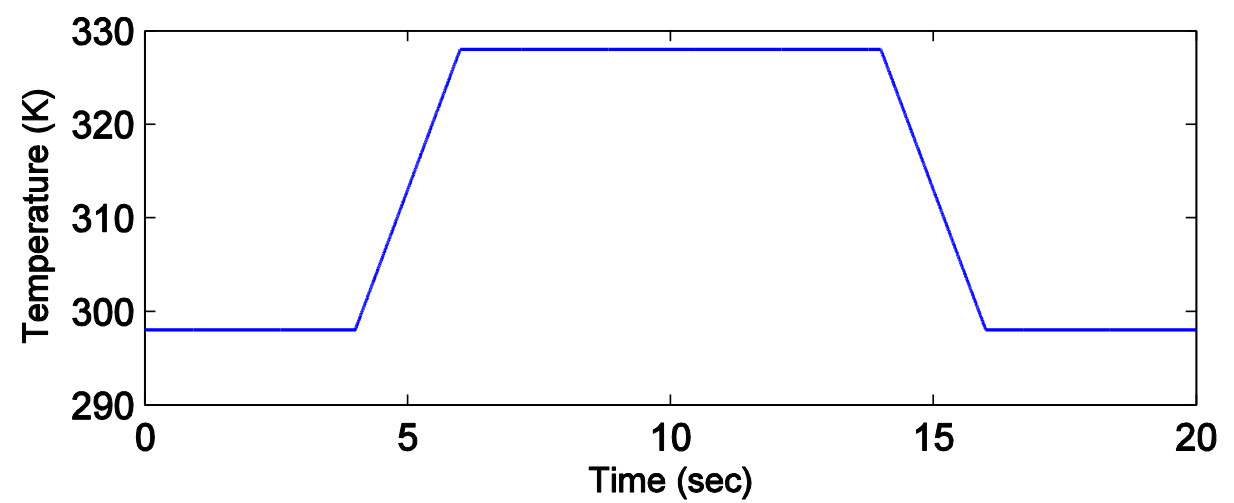

(a)

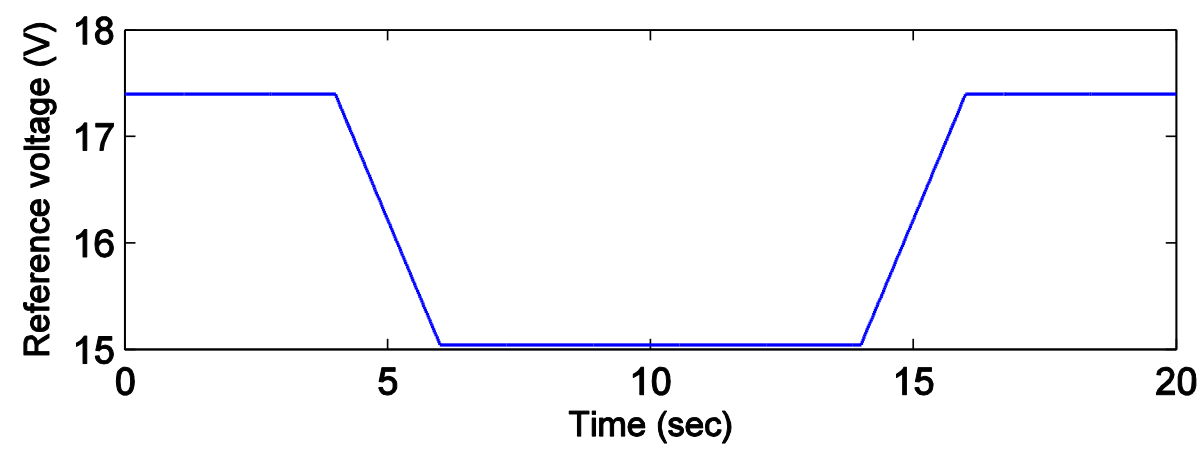

(b)

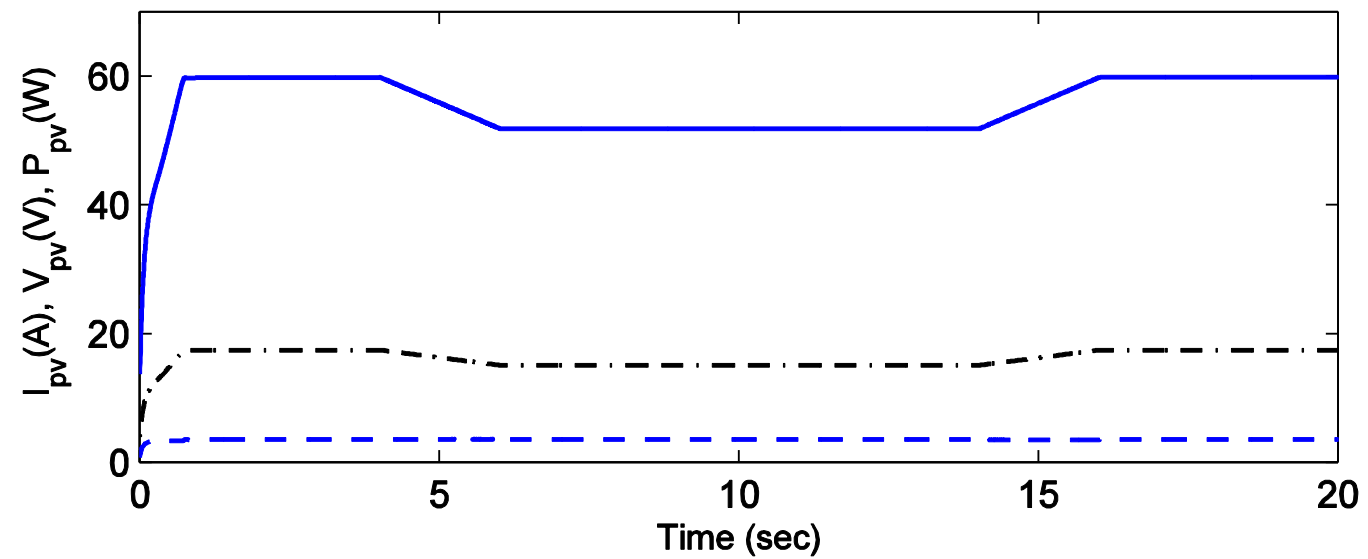

(c)

Fig. 9. Applying the ASMC in case 2, (a) Temperature profile, (b) Maximum power voltage $v_{p v}^{*}$, and (c) PV current $i_{p v}(--)$, PV voltage $v_{p v}(-$.$) , and PV generated power P_{p v}(-)$. 


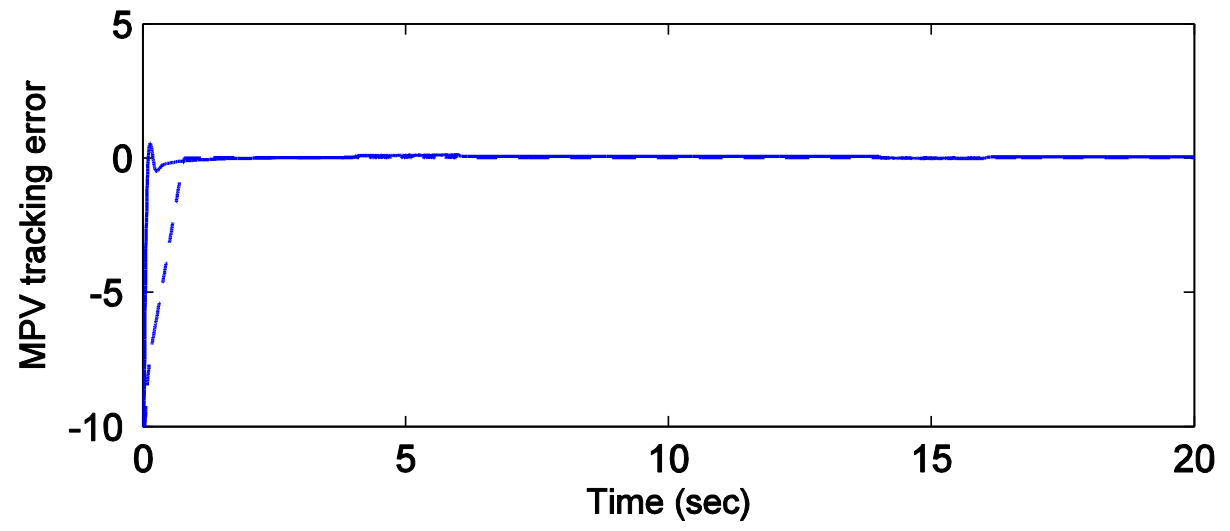

(a)

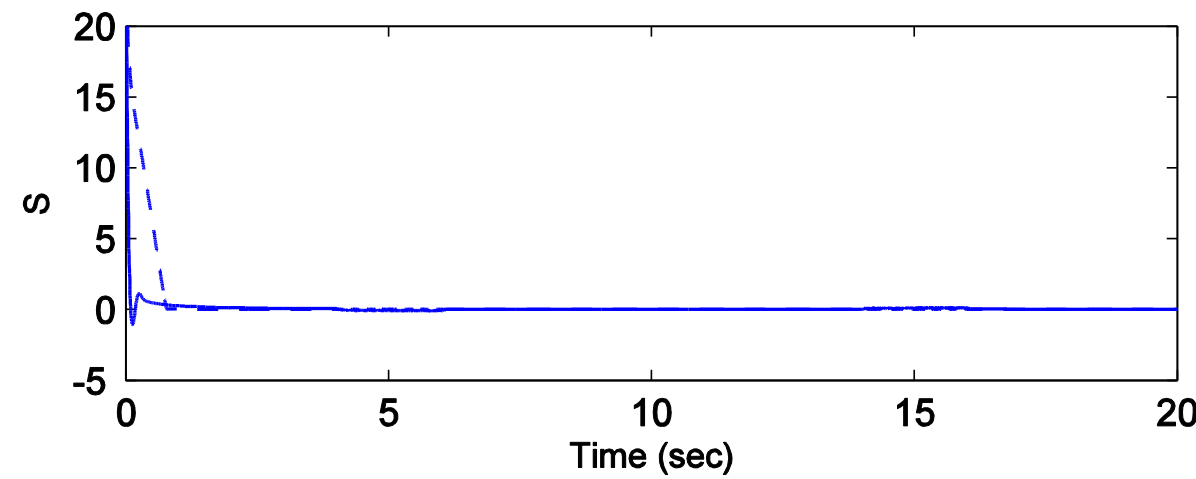

(b)

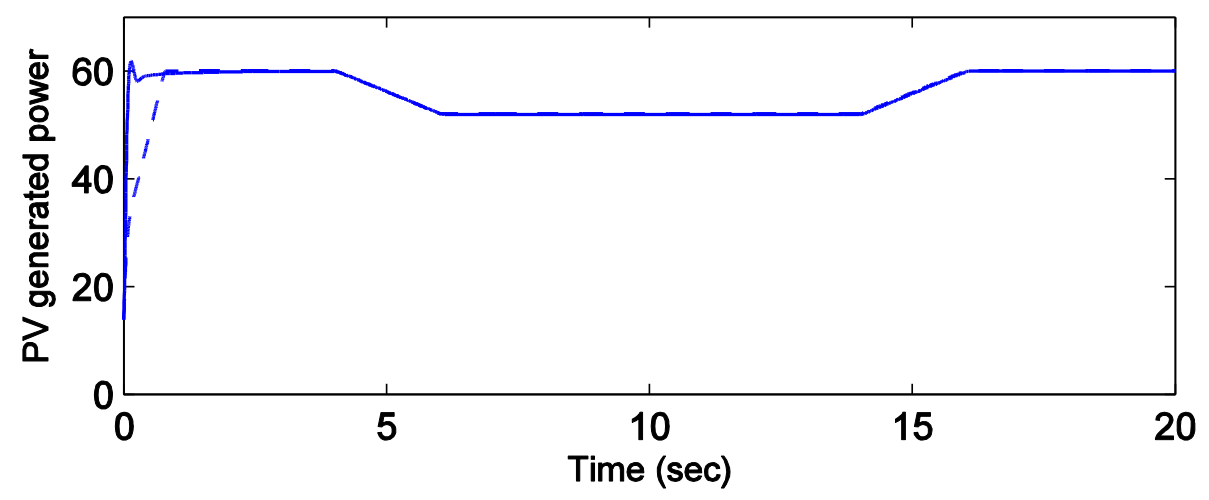

(c)

Fig. 10. Time responses under varying temperature, obtained by applying the ASMC (- -) and the adaptivebased $H_{\infty}$ tracking control (-), (a) MPV tracking error $e_{1}$, (b) Error metric function $S$, and (c) PV generated power $P_{p v}$. 


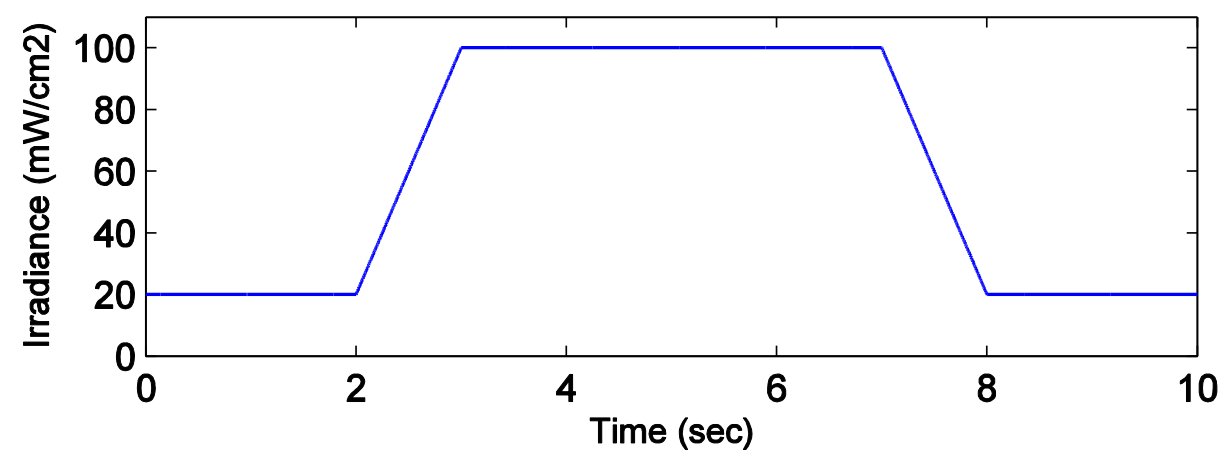

(a)

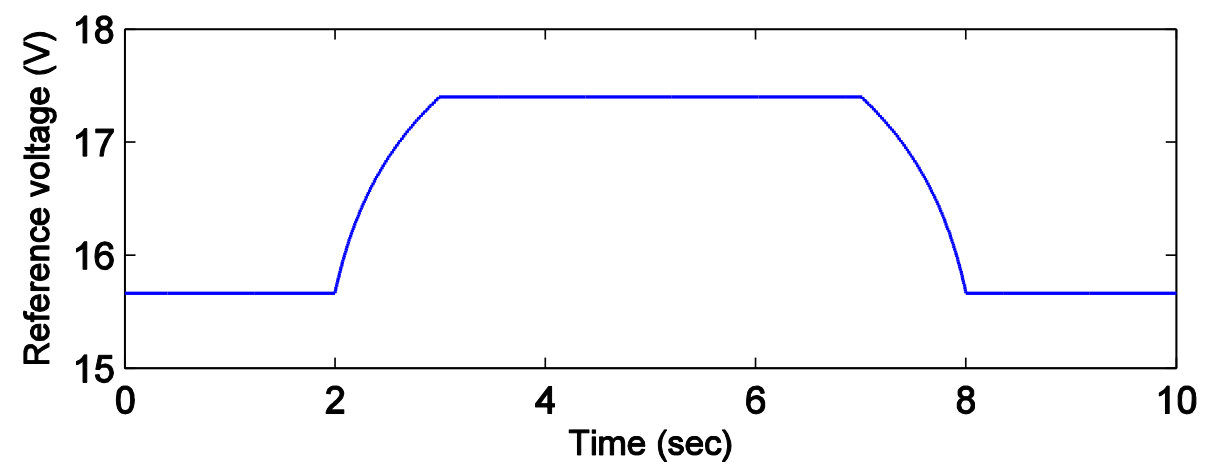

(b)

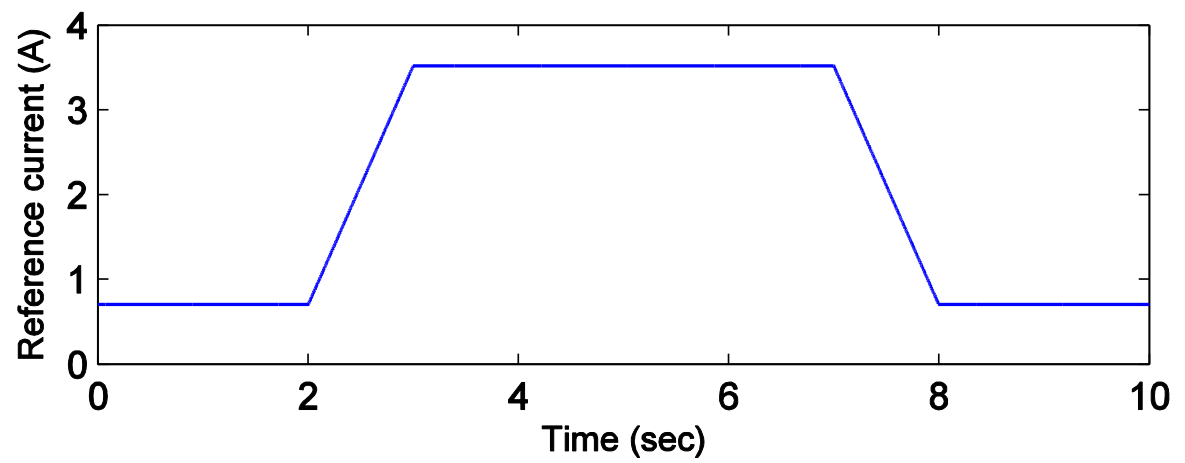

(c)

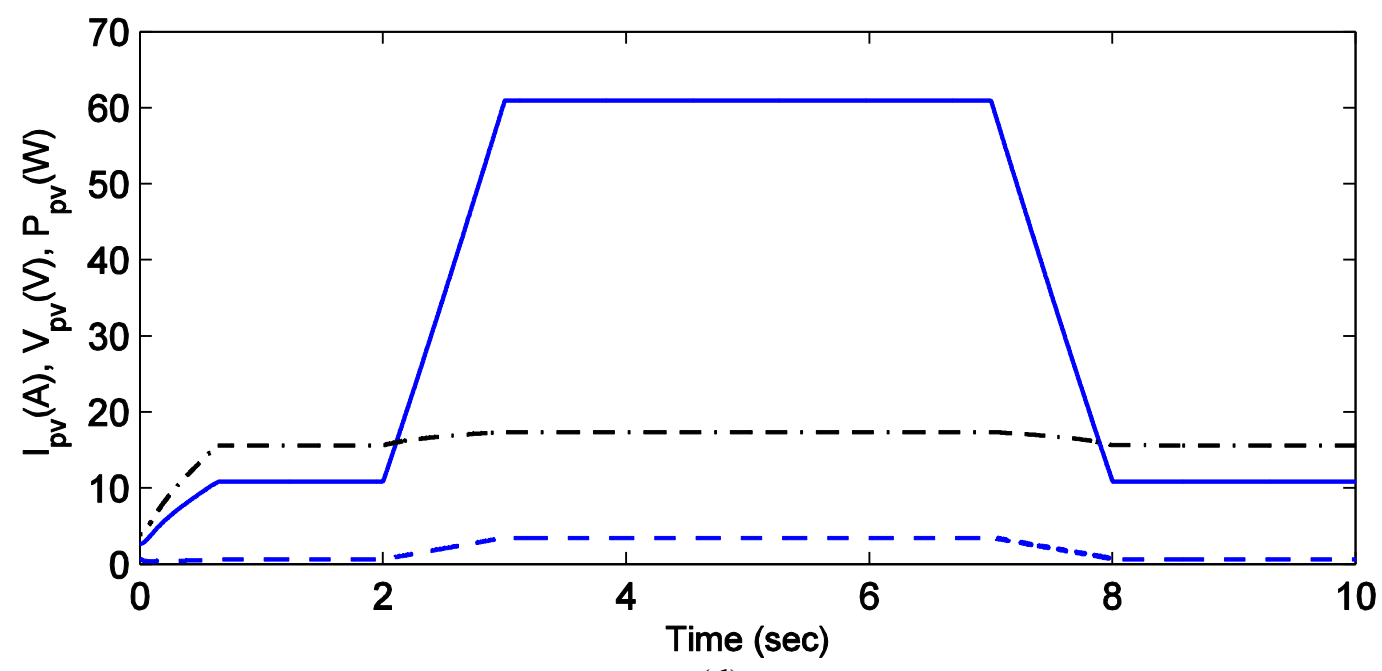

(d)

Fig. 11. Applying the ASMC in case 3, (a) Irradiance profile, (b) Maximum power voltage $v_{p v}^{*}$, (c) Reference current $i_{L}^{*}$, and (d) PV current $i_{p v}(--)$, PV voltage $v_{p v}\left(-\right.$.), and PV generated power $P_{p v}(-)$. 


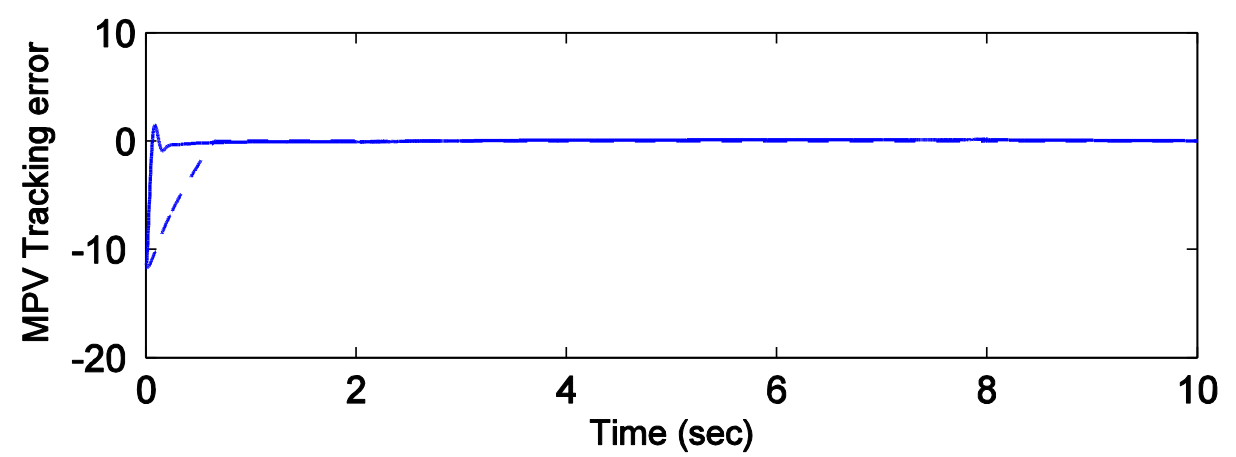

(a)

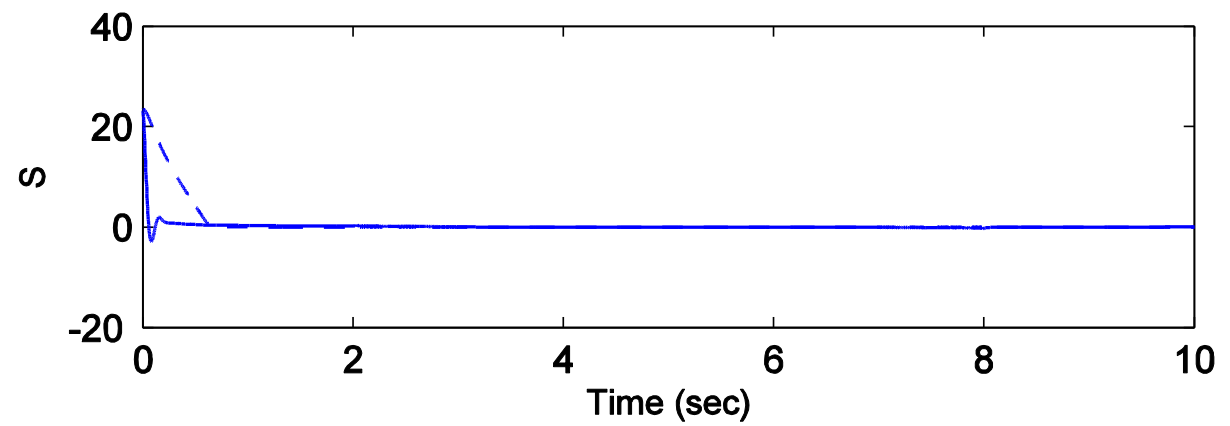

(b)

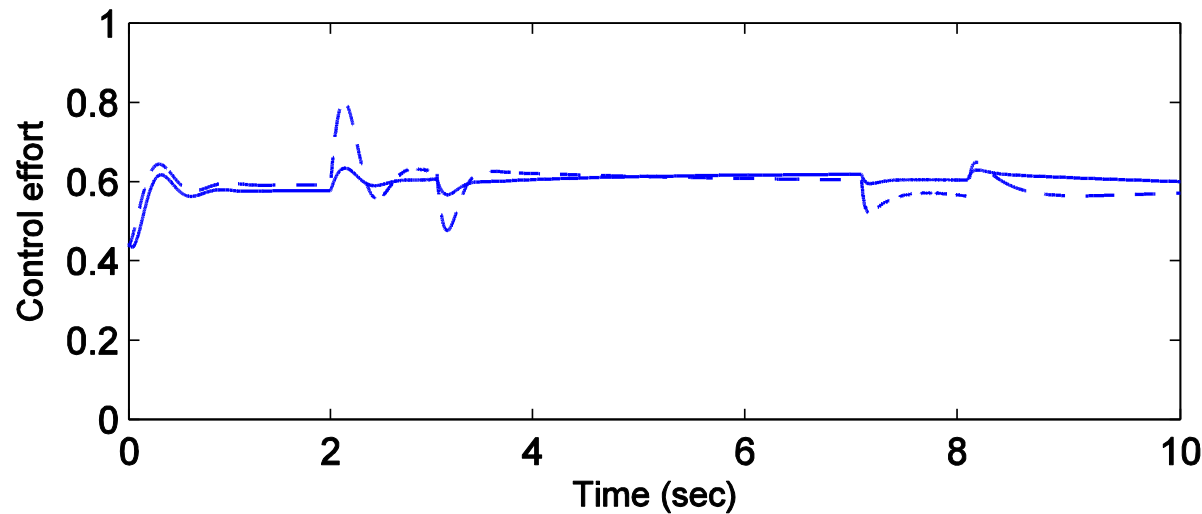

(c)

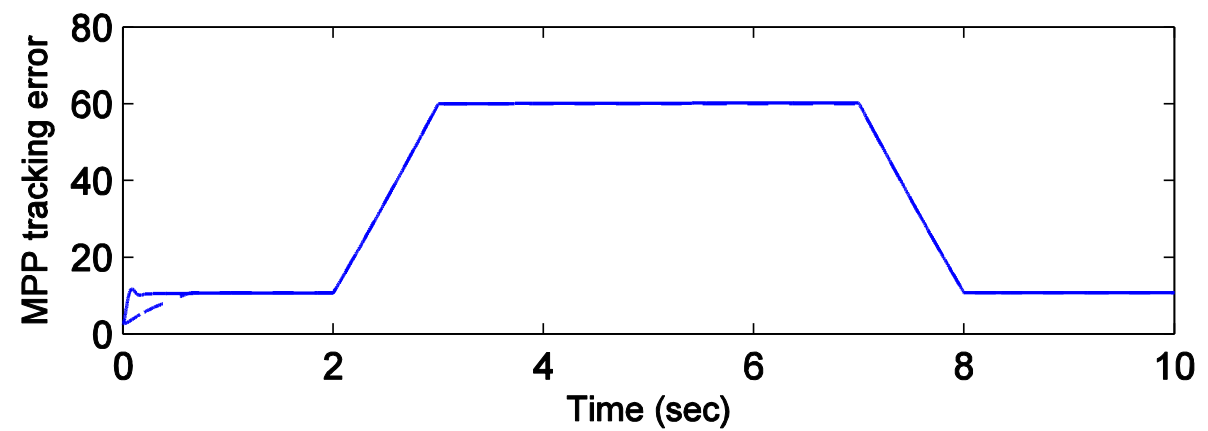

(d)

Fig. 12. System responses under varying irradiance, obtained by applying the ASMC (- -) and the adaptivebased $H_{\infty}$ tracking control (-), (a) MPV tracking error $e_{1}$, error metric function $S$, (c) Duty cycle $u(t)$, and (d) PV generated power $P_{p v}$. 


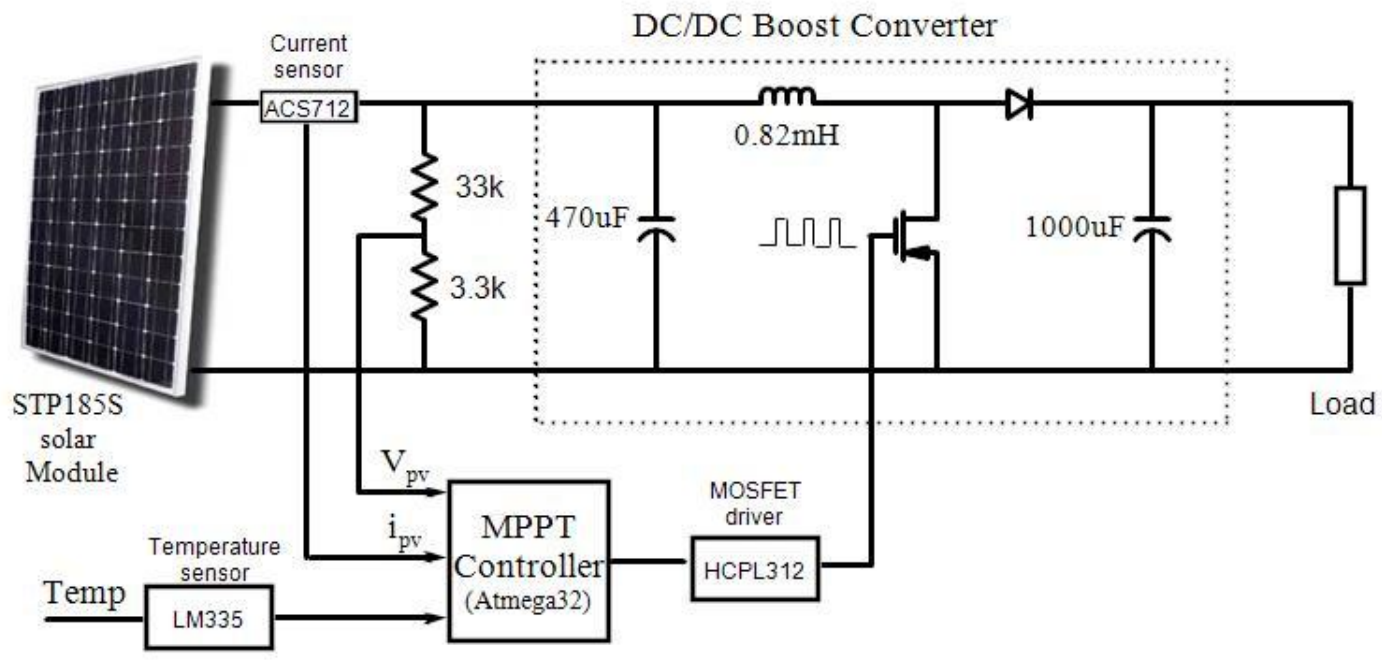

(a)

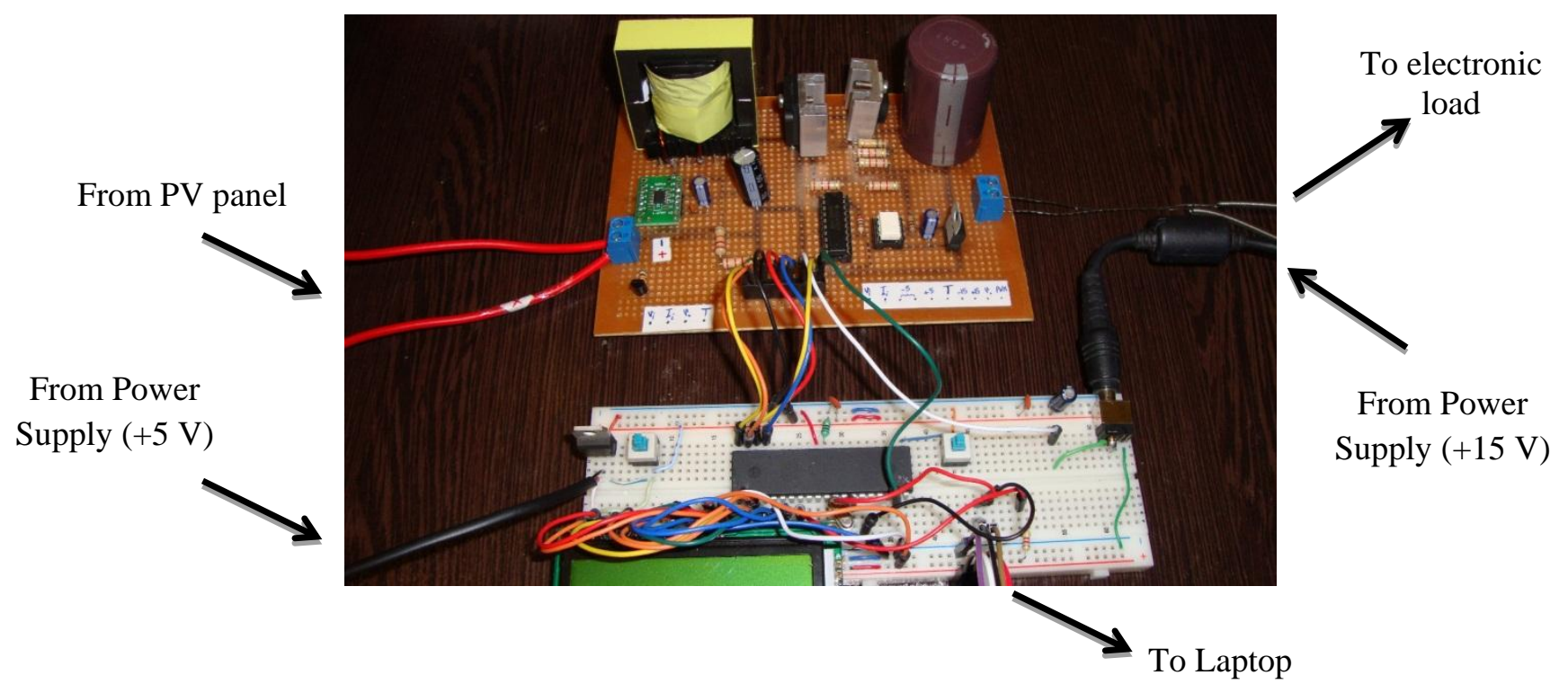

(b)

Fig. 13. Experimental configuration, (a) Block diagram, and (b) Boost converter and the circuit accessories 


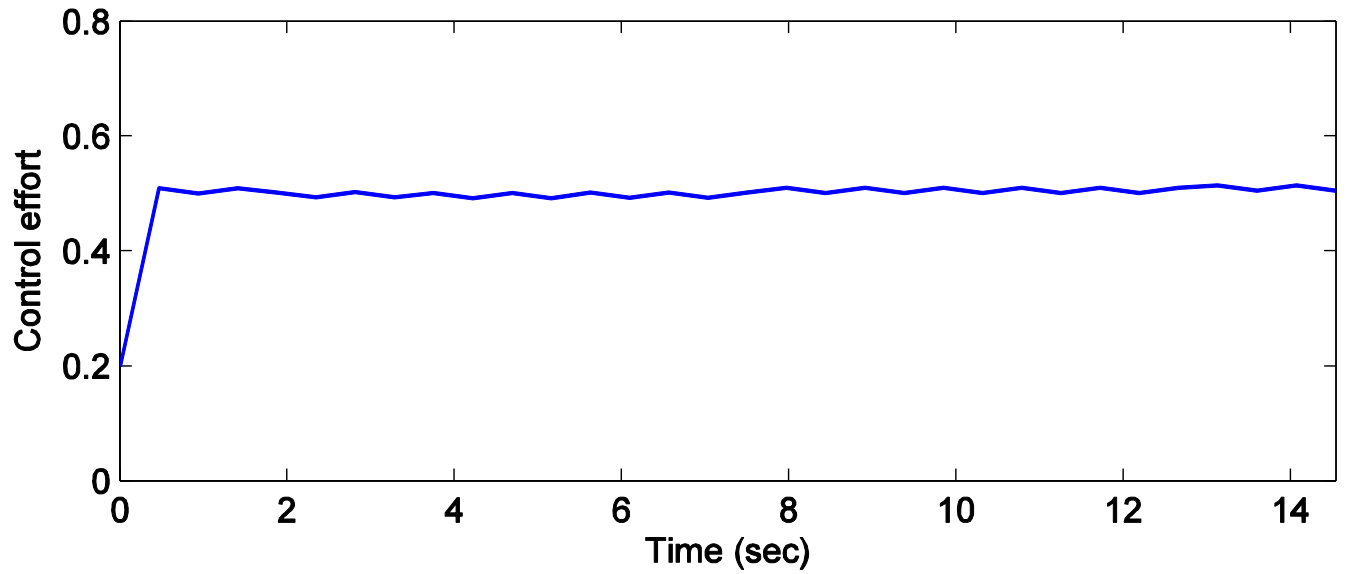

(a)

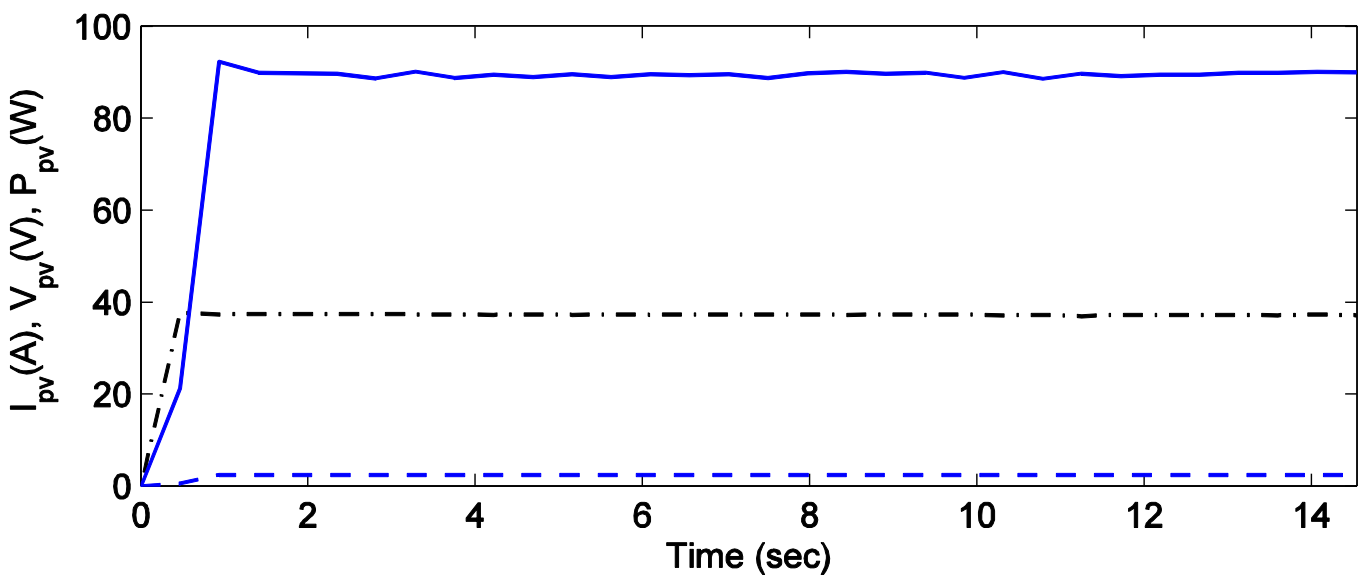

(b)

Fig. 14. Experimetal results of applying ASMC, (a) Control input $u(t)$, and (b) PV current $i_{p v}(--)$, PV voltage $v_{p v}(-$.$) , and PV generated power P_{p v}(-)$. 


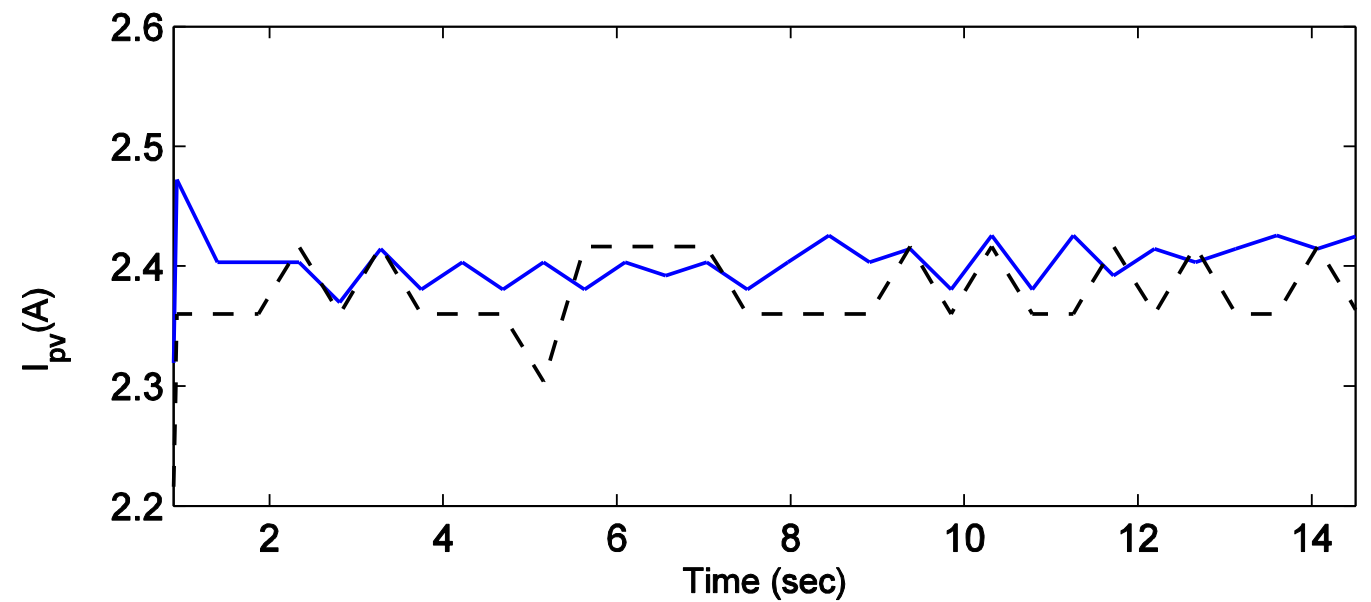

(a)

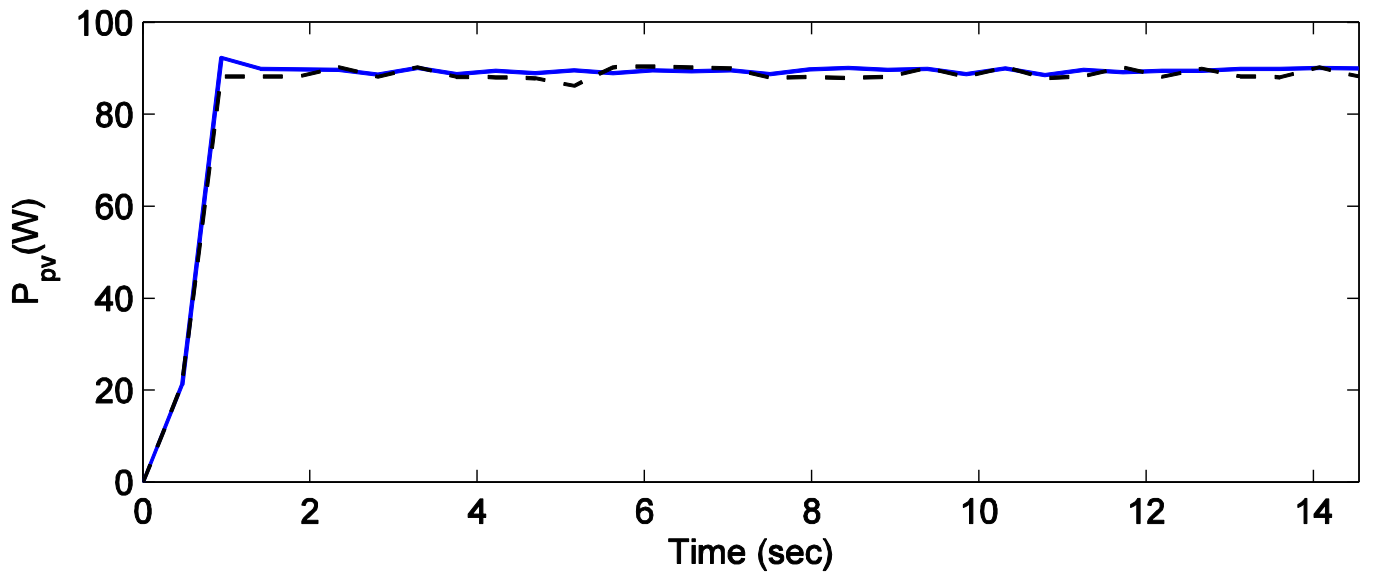

(b)

Fig. 15. Experimental verification by applying the proposed ASMC (-) and the P\&O algorithm (- -), (a) PV current $i_{p v}$, and (b) Generated power $P_{p v}$. 